ORMOND, J.K. (1948) Bilateral ureteral obstruction due to envelopment and compression by an inflammatory retroperitoneal fibrosis. Journal of Urology, 19, 1072.

ORMOND, J.K. (1966) Idiopathic retroperitoneal fibrosis: an established clinical entity. Journal of American Medical Association, 174, 1661.

Patterson, R.J., Bickel, M.H. \& Dahlin, D.C. (1964) Idiopathic avascular necrosis of the head of femur. Journal of Bone and Joint Surgery, 46a, 267.
Phemister, D.B. (1934) Fractures of the neck of femur producing aseptic necrosis of the head of femur. Surgery, Gynaecology and Obstetrics, 159, 415.

Que, G.S. \& MAlldema, E. (1964). A case of retroperitoneal fibrosis presenting as a systemic collagen disease. American Journal of Medicine, 36, 220.

TRUETA, J. \& HARRISON, M.H.M. (1953) The normal vascular anatomy of the femoral head in ault men. Journal of Bone and Joint Surgery, 35b, 442.

\title{
An unexpected abdominal mass
}

\author{
MEIRION THOMAS* \\ M.B., B.S. \\ Masaka Hospital, Uganda
}

\begin{abstract}
Summary
An unusual complication of ectopic pregnancy, presenting during post-menopausal life, is reported. As hospital services become available to previously medically isolated communities, early diagnosis and treatment will make such cases as this rare.
\end{abstract}

\section{Case report}

A Baganda woman was admitted in February, 1971, complaining of a painless abdominal mass present for over 20 years. She had not menstruated for over 2 years but, like most women in rural Africa, was uncertain of her age. She was probably 50 years old. Of her three children, two had died in the first week of life, and only the third, a 30-year-old male, was alive and well. One year after the birth of her third child, she had had amenorrhoea for 6 months and she thought she was pregnant again. Uneventfully normal menstruation was resumed and she was subsequently sterile.

On abdominal examination, a very large mass, the size of a 20 week pregnancy, was palpated, arising centrally from the pelvis. On vaginal examination, the site of the mass was confirmed and was thought to be in continuity with the uterus. A preoperative diagnosis of calcified fibroids was made.

At laparotomy, the uterus was only slightly enlarged and studded with very small calcified fibroids. The right ovary was atrophic and there was a small right hydrosalpinx. Replacing the left ovary there was an oval, calcified mass, $20 \times 10 \mathrm{~cm}$, attached to the uterus by the left fallopian tube and

\footnotetext{
* Present address: Department of Surgery, Royal Postgraduate Medical School and Hammersmith Hospital, Ducane Road, London, W12 OHS.
}

left utero-ovarian ligament. The mass was densely adherent to small bowel and omentum. Adhesions were divided and a total hysterectomy and bilateral salpingo-oophorectomy was performed.

After operation, the mass was opened using a variety of orthopaedic instruments. The calcified capsule was 1-2 cm thick (Fig. 1). Some foetal parts were identified and removed (Fig. 2), but most of the skeleton had been incorporated into the capsule.

\section{Comment}

It was surprising to us that the patient denied any symptoms referable to a ruptured ectopic pregnancy.

The bone development suggested that the gestational age of the foetus was 5-6 months. Remarkably, this 'missed' ectopic had organized, and over a

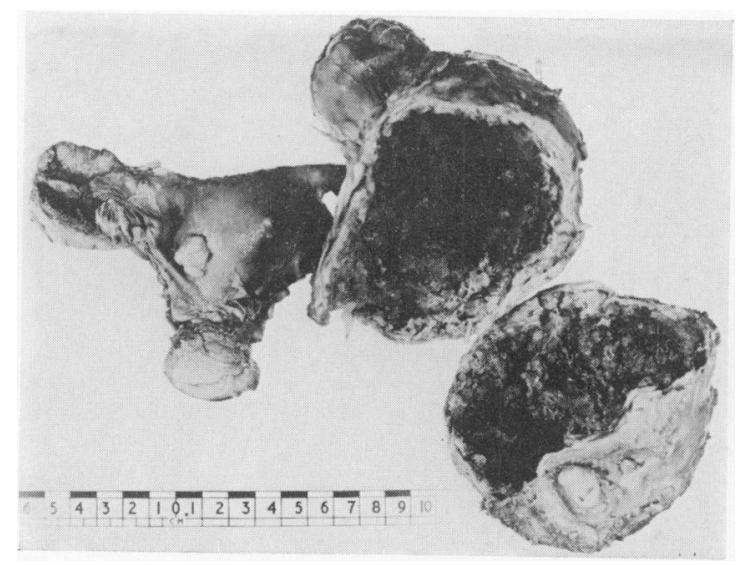

FIG. 1. Operative specimen described in the text. 


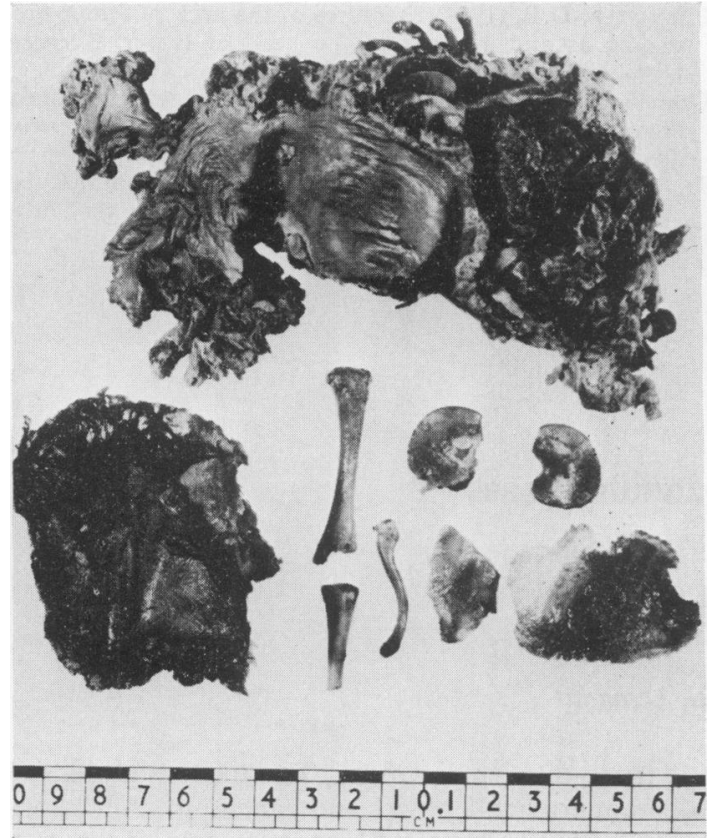

FIg. 2. Foetal parts removed from the tumour. Lower left, scalp; lower right, tibiae, clavicle, iliac bones, skull bones; top, trunk lying horizontally. Diaphragm seen at junction of right and middle third. Below the diaphragm, the heart, lung, pleural cavity and ribs are seen. The remainder of the specimen is skin. period of about 30 years, had developed a formidable calcified capsule. Because the mass replaced and occupied the position of the left ovary, it may well be that this was originally an ovarian ectopic. Spiegelberg's criteria for the diagnosis of an ovarian pregnancy require:

(i) that the tube on the affected side must be intact;

(ii) that the foetal sac must occupy the position of the ovary;

(iii) that the ovary and sac must be connected to the uterus by the utero-ovarian ligament;

(iv) that definite ovarian tissue must be present in the sac wall.

These criteria may have existed originally but were modified by the long process of organization.

It is also interesting that the patient should suddenly decide to attend hospital having had a symptomless abdominal mass for 20 years. Until recent years it was found that patients with chronic gynaecological complaints avoided attending hospital because of fear, prejudice and superstition. With the breakdown of these barriers, the rising popularity of the gynaecology out-patients, and the prompt attendance to hospital, cases like this will become rare, even in rural Africa.

\section{References}

NovaK, E.R. \& WoodRufF, J.D. (1962) Speigelberg's criteria. In: Novak's Gynaecological and Obstetric Patho logy, 5th edn. W. B. Saunders \& Co., Philadelphia and London. 